\title{
Aplicação de insulina passo a passo: construção de vídeos educativos para pacientes e cuidadores
}

\author{
Step-by-step insulin application: making educational videos for patients and caregivers \\ Aplicación de insulina paso a paso: construcción de vídeos educativos para pacientes y cuidadores
}

\author{
Janaina Pereira da Silva ${ }^{1}$ (1) \\ Filipe Andrade Bernardi ${ }^{2}$ (C) \\ Juliana Constantino Franzon ${ }^{1}$ (1) \\ Leonardo Orlandin ${ }^{1}$ (1) \\ Gustavo Zanelatto Ferlin ${ }^{2}$ (C) \\ Gerson Alves Pereira Júnior ${ }^{3}$ (I)
}

1. Universidade de São Paulo, Escola de

Enfermagem de Ribeirão Preto. Ribeirão

Preto, SP, Brasil.

2. Universidade de São Paulo, Faculdade de Medicina de Ribeirão Preto. Ribeirão Preto, SP, Brasil.

3. Universidade de São Paulo, Faculdade de Medicina de Bauru. Bauru, SP. Brasil.

Autor correspondente:

Janaina Pereira da Silva.

E-mail: janaina.pereira.silva@usp.br.

Recebido em 15/02/2020.

Aprovado em 03/06/2020.

DOI:https://doi.org/10.1590/2177-9465-EAN-2019-0343

\section{RESUMO}

Objetivo: descrever o processo de construção, avaliação e adequação de vídeos educativos sobre aplicação de insulina direcionados a pacientes adultos e cuidadores. Método: estudo metodológico, de caráter descritivo, realizado em três fases: construção, avaliação e adequação. A construção dos vídeos foi realizada com base em revisão de literatura e no protocolo do município. A avaliação foi realizada com experts, por meio de um instrumento construído pelos pesquisadores. O nível de concordância entre os experts foi avaliado pelo índice de validade do conteúdo, que orientou o processo de adequação do material. Resultados: os vídeos foram construídos contemplando os principais pontos críticos relacionados à aplicação de insulina. Os itens que obtiveram índice de validade de conteúdo $<0,8$ foram reformulados. Conclusão e Implicações para a prática: foram produzidos dois vídeos que abordam o transporte, armazenamento, preparo e aplicação da insulina, descarte de perfurocortantes e monitorização da glicemia. Os vídeos educativos construídos podem ser considerados ferramentas facilitadoras do processo de educação em diabetes mellitus e úteis na uniformização de orientações. A descrição do processo de construção, avaliação e adequação pode encorajar outros profissionais a desenvolver materiais que respondam às necessidades de seus contextos de trabalho e assim melhorar e qualificar o cuidado às pessoas.

Palavras-chave: Insulina; Diabetes Mellitus; Educação; Pacientes; Filme e Vídeo Educativos.

\section{ABSTRACT}

Objective: to describe the process of development, evaluation, and adaptation of educational videos on insulin application whose target audience was adult patients and caregivers. Methods: descriptive and methodological study, carried out in three phases: development, evaluation, and adaptation. The development of the videos was based on a literature review and the municipality's protocol. The evaluation was carried out by experts by applying an instrument designed by the researchers, and the level of agreement between the experts was assessed by the content validity index, which guided the material adaptation process. Results: the videos were developed to address the main critical points related to insulin application. The items that obtained a content validity index $<0.8$ were reformulated. Conclusion and implications for practice: two videos were made addressing insulin transport, storage, preparation and application, sharps disposal, and blood glucose monitoring. The educational videos made in the present study can be considered tools that contribute to education in diabetes mellitus and are useful for standardizing guidelines. The description of the development, evaluation, and adaptation process can encourage other professionals to develop materials that meet the needs found in their work contexts and, consequently, improve and qualify the care provided to their patients.

Keywords: Insulin; Diabetes Mellitus; Education; Patients; Educational Films and Videos.

\section{RESUMEN}

Objetivo: describir el proceso de construcción, evaluación y adecuación de vídeos educativos sobre la aplicación de insulina dirigidos a pacientes adultos y cuidadores. Método: estudio de carácter descriptivo realizado en tres fases: construcción, evaluación y adecuación. La construcción de los vídeos se realizó en base a una revisión de la literatura y en el protocolo del municipio. La evaluación se realizó con expertos, utilizando un instrumento construido por los investigadores, el nivel de acuerdo entre los expertos se evaluó mediante el índice de validez de contenido, que guió el proceso de adaptación del material. Resultados: los vídeos fueron construidos cubriendo los principales puntos críticos relacionados con la aplicación de insulina. Los ítems que obtuvieron un índice de validez de contenido $<0.8$ fueron reformulados. Conclusión e Implicaciones para la práctica: se realizaron dos vídeos que abordan el transporte, el almacenamiento, la preparación y la aplicación de insulina, la eliminación de objetos punzantes y el control de la glucosa en sangre. Los vídeos educativos construidos pueden considerarse herramientas que facilitan el proceso de educación en diabetes mellitus y útiles para estandarizar las pautas. La descripción del proceso de construcción, evaluación y adaptación puede alentar a otros profesionales a desarrollar materiales que respondan a las necesidades de sus contextos de trabajo y así mejorar la atención a las personas.

Palabras clave: Insulina; Diabetes Mellitus; Educación; Pacientes; Película y Video Educativos. 


\section{INTRODUÇÃO}

A insulina é a medicação indicada para o tratamento de pacientes com diabetes mellitus tipo 1, mas também para 0 tratamento de pacientes com diabetes mellitus tipo 2, quando os alvos terapêuticos não são alcançados com o uso de medicação via oral. ${ }^{1}$

A técnica de aplicação de insulina é um ponto crítico para o controle do diabetes mellitus. Seu domínio pelo paciente e/ou cuidador é tão importante quanto a adequação da prescrição médica às necessidades do paciente. ${ }^{2}$ A equipe de saúde precisa dispor de recursos para capacitar o paciente e o cuidador para a aplicação de insulina. ${ }^{3}$

A via indicada para a administração de insulina pelo paciente é a subcutânea, em função da absorção gradativa que ela possibilita. ${ }^{3} \mathrm{~A}$ aplicação via oral, mais conveniente e desejável, ainda é um desafio para a ciência, que atualmente busca o desenvolvimento de tecnologias que impeçam que a insulina seja degradada pelas enzimas digestivas. ${ }^{4}$

É comum que pacientes com diabetes mellitus adotem comportamentos resistentes em relação ao uso de insulina, por se tratar de uma medicação injetável, associada à dor, ao medo, à limitação da liberdade e ao reconhecimento público acerca do diagnóstico. ${ }^{5}$

Estudos apontam a existência de muitos problemas em relação à técnica de aplicação, fato que destaca a necessidade premente de concentrar esforços na educação do paciente para a autoaplicação e/ou do cuidador, a fim de assumir essa responsabilidade junto ao paciente..$^{6,7}$

A educação em diabetes mellitus tem impacto na redução do risco de complicações agudas e na prevenção de complicações crônicas. ${ }^{1}$ Estudos mostram quedas dos níveis de hemoglobina glicada em pacientes submetidos a intervenções educativas. ${ }^{8,9}$

Nesse sentido, materiais educativos precisam ser incorporados à rotina de cuidados do paciente com diabetes. ${ }^{10}$ Eles são ferramentas que dão suporte aos profissionais na interação com o paciente, permitindo, de maneira fácil e simplificada, a construção do conhecimento. ${ }^{11}$

Dentre os materiais utilizados nas ações educativas junto ao paciente, os recursos visuais e audiovisuais têm se destacado por promoverem maior absorção de conteúdo, além de se caracterizarem como uma prática de desenvolvimento simples, de baixo custo, favorecendo a acessibilidade. ${ }^{12}$ Esses recursos permitem, também, o alívio da carga do profissional de saúde enquanto único provedor de informações, ${ }^{13}$ minimizando a possibilidade de omissão não intencional de aspectos relevantes do cuidado.

A Portaria do Ministério da Saúde oㅜ 2.583 de 10 de outubro de $2007^{14}$, que trata sobre as medicações fornecidas no âmbito do Sistema Único de Saúde para pacientes com diabetes mellitus, enfatiza a necessidade de investimentos no desenvolvimento de ações educativas, no âmbito individual e coletivo, com foco no autocuidado.

Nesse sentido, este estudo tem como objetivo descrever o processo de construção, avaliação e adequação de vídeos educativos sobre aplicação de insulina, direcionados a pacientes adultos e cuidadores.

\section{MÉTODO}

Trata-se de um estudo metodológico, desenvolvido em três fases, de caráter descritivo, sobre a construção, avaliação e adequação de vídeos educativos sobre a aplicação de insulina.

A construção dos vídeos foi norteada pelo trabalho publicado por Echer, que trata das etapas do processo de construção de manuais didáticos para o cuidado em saúde. Apesar de Echer ter discorrido sobre a construção de manuais, e o presente estudo abordar a construção de vídeos, as orientações aportadas por essa autora direcionaram a condução desse trabalho. Em seu trabalho Echer, traz uma série de orientações pertinentes e didáticas para a construção de materiais educativos, como a importância da transformação da linguagem técnica em linguagem acessível ao público-alvo, os critérios de seleção do conteúdo a ser incluído no material e a qualificação do material por meio da avaliação de especialistas. ${ }^{15}$

\section{Fase 1 - Construção do vídeo educativo}

Foi realizado levantamento bibliográfico sobre a aplicação de insulina e a produção e validação de materiais educativos. Por conseguinte, o Protocolo de Atendimento em Hipertensão e Diabetes do município onde o estudo foi desenvolvido foi adotado como referência para a construção dos vídeos. ${ }^{16}$ As evidências científicas divergentes ao protocolo foram discutidas junto à equipe coordenadora do Programa de Atenção às Pessoas com Doenças Crônicas Não Transmissíveis.

A princípio, foi construído apenas um vídeo, contemplando as informações essenciais para o desenvolvimento do procedimento de aplicação de um e de dois tipos de insulina na mesma seringa Esse vídeo foi elaborado com ênfase no esclarecimento dos pontos críticos mais prevalentes descritos na literatura e identificados na prática diária dos pesquisadores. Para tanto, foi realizada a elaboração de um roteiro que direcionou a construção do vídeo.

O roteiro elaborado para a construção do vídeo foi dividido em duas colunas: a primeira, referente ao áudio, e a segunda, referente à imagem. Para cada áudio foi atribuída uma imagem correspondente. O áudio foi composto por trilha sonora e narração, e a imagem, por vídeo, foto, desenho e animação. Após atribuição de áudios e imagens, foram realizadas revisões cronometradas do áudio, a fim de produzir um material educativo com tempo de duração e velocidade adequados às necessidades do público-alvo, sem prejuízo para a abordagem do conteúdo, para a compreensão e para a manutenção da atenção. A captação das imagens e do áudio foi realizada em um estúdio, por um profissional com formação na área de som e imagem, que também realizou a edição do material.

O conteúdo abordado no vídeo inicial foi organizado em passos, a fim de facilitar a organização do material e a compreensão do público-alvo.

\section{Fase 2 - Avaliação do vídeo educativo}

Após o processo de construção, o vídeo inicial produzido foi submetido à avaliação de um grupo de experts em diabetes mellitus e doenças crônicas não transmissíveis. A seleção 
dos experts foi feita por conveniência, a partir da análise do Currículo Lattes.

A avaliação do vídeo foi desenvolvida em um único encontro, com duração de três horas, no qual foi feita a explanação acerca do projeto de pesquisa, a exposição do material desenvolvido, a discussão multiprofissional e o preenchimento dos instrumentos de avaliação. Na avaliação, o vídeo foi apresentado utilizando-se um aparelho multimídia e caixa de som. Os experts discutiram o material produzido sob uma perspectiva multiprofissional. Todos os passos do vídeo foram abordados e as sugestões resultantes foram registradas pela equipe de pesquisadores presentes no processo de avaliação.

O instrumento de avaliação utilizado foi construído pelos pesquisadores, contendo 28 itens relevantes referentes ao conteúdo e à aparência do vídeo. O instrumento foi elaborado com afirmações e possibilidades de resposta tipo likert, com os seguintes níveis de concordância: 4 = adequado, 3 = adequado com possibilidade de revisão, 2 = precisa ser reformulado e 1 = inadequado. Os itens que receberam pontuação "1" e "2" foram submetidos a adequações. Já os itens que receberam pontuação "3" e "4" foram mantidos. Para avaliar a concordância entre os juízes, utilizou-se o índice de validade de conteúdo (IVC), que é calculado a partir da seguinte fórmula: número total de respostas "3" e "4" dividido pelo número total de respostas. O índice de concordância considerado satisfatório foi $\geq 0,8 .{ }^{17,18}$ Os experts também preencheram um instrumento de caracterização sócio-acadêmico-profissional.

\section{Fase 3 - Adequação do vídeo pós avaliação}

A partir das avaliações resultantes da fase 2, os itens do vídeo inicial que obtiveram IVC $<0,8$ foram reformulados. As considerações dos experts que não estavam contempladas no instrumento de avaliação também foram consideradas.

A avaliação resultou na produção de dois vídeos: um contendo o processo de preparação de apenas um tipo de insulina, e outro referente ao processo de preparação de dois tipos de insulina na mesma seringa. Para tanto, o roteiro inicial foi transformado em dois roteiros e foi realizada nova captação de áudio e imagem. Essa etapa contou com a colaboração de um profissional da área de produção audiovisual.

Este estudo foi aprovado pelo Comitê de Ética em Pesquisa da Escola de Enfermagem de Ribeirão Preto da Universidade de São Paulo, sob o parecer consubstanciado nํㅜ 2.144.495. Foram atendidos os fundamentos contidos na Resolução CNS 466/2012, que trata sobre os aspectos éticos da pesquisa envolvendo seres humanos. Para a participação dos sujeitos, foi utilizado termo de Consentimento Livre e Esclarecido.

\section{RESULTADOS}

\section{Fase 1 - Construção do vídeo educativo}

Na primeira fase, foi construído um único vídeo, intitulado: "Aplicação de insulina: passo-a-passo". Ele foi construído com foco em orientações direcionadas a pacientes e/ou cuidadores que utilizam seringa para aplicação, já que, no município onde o estudo foi desenvolvido, a administração de insulina com outros dispositivos, como caneta e bomba de infusão contínua, ainda é incipiente.

Esse vídeo foi produzido com tempo de duração de 08 minutos e 34 segundos e foi dividido em 13 passos para facilitar a organização do conteúdo: 1-Como transportar insulina, 2-Onde guardar a insulina, 3-Prazo de validade da insulina após abertura do frasco, 4-Materiais necessários para realizar a aplicação, 5-Áreas do corpo onde a insulina pode ser aplicada, 6-Como organizar a aplicação, 7-Como preparar a insulina para aplicação, 8-Como preparar dois tipos de insulina na mesma seringa, 9-Como realizar a aplicação, 10-Reutilização de seringas, 11-Descarte das seringas, 12-Monitoramento da glicemia e 13-Sinais e sintomas de hipoglicemia. Apesar de reconhecer a existência de outros pontos críticos, foram mantidos os passos descritos acima para construção do material.

\section{Fase 2 - Avaliação do vídeo educativo}

Entre os $10(100,0 \%)$ experts que avaliaram o material, encontravam-se $03(30,0 \%)$ médicos endocrinologistas, $03(30,0 \%)$ enfermeiras, 01 (10,0\%) farmacêutica, 01 (10,0\%) dentista, $01(10,0 \%)$ psicóloga e 01 (10,0\%) médica clínica geral, todos com ampla experiência no cuidado ao paciente com diabetes mellitus em uso de insulina.

Em relação à atuação profissional dos experts, 01 (10,0\%) ocupava o cargo de coordenador do Programa de Doenças Crônicas Não Transmissíveis do município onde o estudo foi desenvolvido, 01 (10,0\%) atuava como gerente de um ambulatório de especialidades, 01 (10,0\%) atuava na docência e 07 (70,0\%) atuavam na assistência direta aos pacientes. A presença de experts vinculados à gestão permitiu a discussão do material produzido à luz dos aspectos financeiros, gerenciais e burocráticos que tangem a assistência, assim como a presença de profissionais da assistência permitiu a discussão dos problemas e dificuldades vivenciados no dia-a-dia.

Em relação à potencialidade para o alcance dos objetivos educacionais que visam a resolução dos problemas identificados na assistência e na literatura, o vídeo teve 15 itens avaliados pelos experts. Entre os itens avaliados, 14 (93.3\%) obtiveram IVC $\geq 0,8$. $\mathrm{O}$ item referente à capacidade que o vídeo tem em favorecer a compreensão da técnica de mistura de insulinas obteve IVC 0,7. A reformulação desse item foi sugerida por 03 (30\%) dos experts, conforme pode ser observado na Tabela 1.

Em relação aos aspectos técnicos e organizacionais do vídeo, 10 (100,0\%) dos itens avaliados alcançaram IVC $\geq 0,8$. Todavia, conforme pode ser observado na Tabela 2 , a qualidade do som foi considerada inadequada por 01 (10,0\%) dos experts.

\section{Fase 3 - Adequação do vídeo pós avaliação}

Frente à avaliação dos experts e análise do IVC, foram realizadas as adequações necessárias.

O item que avaliava a capacidade do vídeo em favorecer a compreensão da técnica de mistura de insulinas obteve IVC $=0,7$ e $03(30 \%)$ dos experts sugeriram a reformulação dessa parte do vídeo. Assim, optou-se por produzir dois vídeos distintos para melhor compreensão: um deles contendo o processo de preparação de apenas um tipo de insulina e outro referente ao 
Tabela 1. Avaliação do vídeo educativo "Aplicação de insulina: passo a passo". Ribeirão Preto - SP, Brasil, 2018. (n=10).

\begin{tabular}{|c|c|c|c|c|c|}
\hline Itens avaliados & Adequado & $\begin{array}{l}\text { Adequado com } \\
\text { possibilidade de } \\
\text { revisão }\end{array}$ & $\begin{array}{l}\text { Precisa ser } \\
\text { reformulado }\end{array}$ & Inadequado & IVC \\
\hline $\begin{array}{l}\text { Favorece a compreensão do transporte adequado da } \\
\text { insulina }\end{array}$ & $08(80,0 \%)$ & $02(20,0 \%)$ & - & - & 1,0 \\
\hline $\begin{array}{l}\text { Favorece a compreensão das condições ideais de } \\
\text { armazenamento }\end{array}$ & $06(60,0 \%)$ & $04(40,0 \%)$ & - & - & 1,0 \\
\hline $\begin{array}{l}\text { Favorece o controle da validade do frasco de insulina após } \\
\text { abertura }\end{array}$ & $07(70,0 \%)$ & $02(20,0 \%)$ & $01(10,0 \%)$ & - & 0,9 \\
\hline $\begin{array}{l}\text { Favorece a compreensão da necessidade de retirada do } \\
\text { frasco de insulina da geladeira 15-30 minutos antes da } \\
\text { aplicação }\end{array}$ & $07(70,0 \%)$ & $02(20,0 \%)$ & 01 (10,0\%) & - & 0,9 \\
\hline $\begin{array}{l}\text { Favorece a compreensão da técnica de homogeneização da } \\
\text { insulina NPH }\end{array}$ & $07(70,0 \%)$ & $03(30,0 \%)$ & - & - & 1,0 \\
\hline $\begin{array}{l}\text { Favorece a compreensão da necessidade de desinfecção da } \\
\text { borracha do frasco de insulina com algodão e álcool }\end{array}$ & $07(70,0 \%)$ & $02(20,0 \%)$ & $01(10,0 \%)$ & - & 0,9 \\
\hline $\begin{array}{l}\text { Favorece a compreensão da escala de graduação das } \\
\text { seringas }\end{array}$ & $03(30,0 \%)$ & $06(60,0 \%)$ & 01 (10,0\%) & - & 0,9 \\
\hline $\begin{array}{l}\text { Favorece a compreensão da necessidade de injeção de ar } \\
\text { no frasco antes da aspiração }\end{array}$ & $06(60,0 \%)$ & $03(30,0 \%)$ & $01(10,0 \%)$ & - & 0,9 \\
\hline $\begin{array}{l}\text { Favorece a compreensão da técnica de mistura de insulinas } \\
\mathrm{NPH} \text { e regular }\end{array}$ & $04(40,0 \%)$ & $03(30,0 \%)$ & $03(30,0 \%)$ & & 0,7 \\
\hline Favorece a identificação das áreas de aplicação da insulina & $07(70,0 \%)$ & $03(30,0 \%)$ & - & - & 1,0 \\
\hline $\begin{array}{l}\text { Favorece a compreensão da organização do rodízio do local } \\
\text { de aplicação }\end{array}$ & $06(60,0 \%)$ & $03(30,0 \%)$ & $01(10,0 \%)$ & - & 0,9 \\
\hline $\begin{array}{l}\text { Favorece a compreensão da necessidade de manutenção } \\
\text { da agulha no tecido subcutâneo por } 5 \text { segundos após a } \\
\text { aplicação }\end{array}$ & $07(70,0 \%)$ & $03(30,0 \%)$ & - & - & 1,0 \\
\hline $\begin{array}{l}\text { Favorece a compreensão do descarte adequado de } \\
\text { perfurocortantes }\end{array}$ & $08(80,0 \%)$ & $02(20,0 \%)$ & - & - & 1,0 \\
\hline Favorece a compreensão da monitorização da glicemia & $06(60,0 \%)$ & $04(40,0 \%)$ & - & - & 1,0 \\
\hline $\begin{array}{l}\text { Favorece o reconhecimento dos sinais e sintomas de } \\
\text { hipoglicemia }\end{array}$ & $07(70,0 \%)$ & $02(20,0 \%)$ & $1(10,0 \%)$ & - & 0,9 \\
\hline
\end{tabular}

processo de preparação de dois tipos de insulina na mesma seringa. O primeiro vídeo com duração de 08 minutos e 09 segundos e o segundo com duração de 08 minutos e 55 segundos.

Apesar da avaliação positiva do vídeo inicial em relação aos aspectos técnicos e organizacionais, optou-se por realizar nova captação, tanto do áudio quanto das imagens, para viabilizar o atendimento das sugestões. A nova captação de imagens foi feita em ambiente domiciliar, a fim de melhorar a contextualização e demonstrar, por meio do vídeo, aspectos relevantes que haviam sido demonstrados por meio de desenho no vídeo produzido inicialmente, como, por exemplo, o armazenamento dos frascos de insulina na geladeira. Além disso, com a nova captação de imagens, foi possível aprimorar aspectos relacionados ao contraste na seleção das cores de fundo e dos objetos, melhorando a nitidez das imagens. Já o áudio, que foi apontado como inadequado por 01 (10\%) expert, foi regravado em outro estúdio.
Adequações relacionadas ao texto também foram feitas, com o objetivo de melhorar a compreensão do procedimento, assim como para a descrição do aspecto dos dois tipos de insulina e a utilização de termos descritivos mais comuns, como insulina leitosa e insulina transparente, foram acrescentadas legendas, como método de reforço da informação fornecida no áudio. Também foram incluídas informações referentes à descrição das partes que compõem a seringa de aplicação de insulina, já que, em vários momentos do vídeo, utilizou-se a palavra "êmbolo" para explicar como realizar alguns procedimentos, e para tal palavra não foi encontrada substituta que pertencesse à linguagem comum do público-alvo.

A Figura 1 representa recortes de diferentes momentos dos vídeos finais produzidos, que se encontram disponíveis para acesso e download nos links:

https://www.youtube.com/watch?v=0_6kV6M--pQ\&t=37s https://www.youtube.com/watch?v=Fnp8AB7dwXc\&t=76s 
Tabela 2. Avaliação dos aspectos técnicos e organizacionais do vídeo "Aplicação de insulina: passo a passo". Ribeirão Preto - SP, Brasil, 2018. $(n=10)$.

\begin{tabular}{llcccc}
\hline \multicolumn{1}{c}{ Itens avaliados } & Adequado & $\begin{array}{c}\text { Adequado com } \\
\text { possibilidade } \\
\text { de revisão }\end{array}$ & $\begin{array}{c}\text { Precisa ser } \\
\text { reformulado }\end{array}$ & Inadequado & IVC \\
\hline Tempo de duração & $07(70,0 \%)$ & $03(30,0 \%)$ & - & - & 1,0 \\
\hline Qualidade do som & $07(70,0 \%)$ & $02(20,0 \%)$ & - & $01(10,0 \%)$ & 0,9 \\
\hline Imagens & $06(60,0 \%)$ & $03(30,0 \%)$ & $01(10,0 \%)$ & - & 0,9 \\
\hline Linguagem & $07(70,0 \%)$ & $02(20,0 \%)$ & $01(10,0 \%)$ & - & 0,9 \\
\hline Clareza e objetividade & $05(50,0 \%)$ & $04(40,0 \%)$ & $01(10,0 \%)$ & - & 0,9 \\
\hline $\begin{array}{l}\text { Organização do conteúdo } \\
\text { Concordância e ortografia }\end{array}$ & $09(90,0 \%)$ & $01(10,0 \%)$ & - & - & 1,0 \\
\hline $\begin{array}{l}\text { As informações apresentadas estão corretas } \\
\text { Coerência do conteúdo com as necessidades } \\
\text { cotidianas do público-alvo }\end{array}$ & $08(60,0 \%)$ & $04(40,0 \%)$ & - & - & 1,0 \\
$\begin{array}{l}\text { Atende aos objetivos de instituições que } \\
\text { atendem pacientes em insulina e seus } \\
\text { cuidadores }\end{array}$ & $07(70,0 \%)$ & $03(30,0 \%)$ & - & - & 1,0 \\
\hline
\end{tabular}

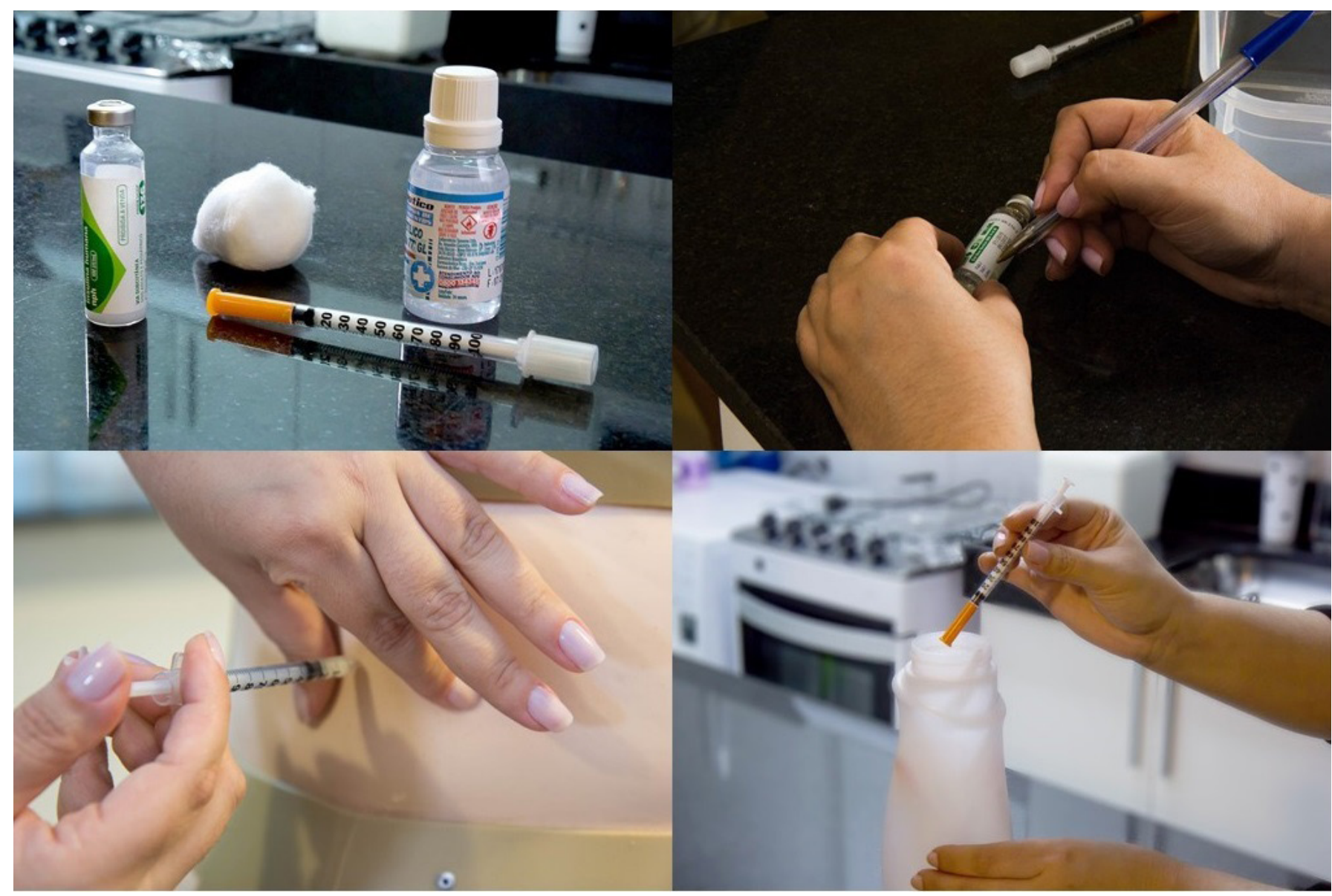

Figura 1. Recortes dos vídeos: "Aplicação de insulina: passo a passo". Ribeirão Preto, SP, Brasil, 2018. Fonte: arquivos do pesquisador 


\section{DISCUSSÃO}

O foco no atendimento ao paciente com diabetes em uso de insulina é, habitualmente, o ajuste de dose frente aos resultados dos exames laboratoriais. ${ }^{19}$

O acesso a materiais educativos é difícil, o que pode desencorajar as equipes de saúde quanto à utilização desses recursos para a capacitação do paciente e do cuidador. ${ }^{11}$ Os materiais educativos disponíveis, em sua maioria, são publicados por corporações como as sociedades de especialidades médicas e grandes empresas. Considerando a grande desigualdade entre as regiões brasileiras, as recomendações contidas nesses materiais muitas vezes não são factíveis, fato que inviabiliza a utilização.

A reutilização de seringas, por exemplo, é uma prática vigente no Brasil. Apesar de a Sociedade Brasileira de Diabetes contraindicar, pelo risco de infecção e lipohipertrofia, o Ministério da Saúde recomenda que a seringa seja reutilizada pelo mesmo paciente no domicílio por até sete vezes, considerando alguns critérios. ${ }^{3,20}$ No município onde este estudo foi desenvolvido, é recomendado que a seringa seja reutilizada pelos pacientes que não fazem mistura de insulinas por no máximo quatro vezes, e para aqueles que fazem mistura de insulinas, por no máximo duas vezes. ${ }^{16}$

Confrontar as orientações do material educativo com as evidências científicas e o protocolo do município, sem prejuízo aos resultados ao paciente, pode ter sido a maior dificuldade na elaboração desse trabalho. Essa dificuldade foi ressaltada por todos os experts durante a avaliação do material. As questões abordadas pelos experts neste estudo foram relevantes e consideradas pelos pesquisadores, o que contribuiu sobremaneira para a maior qualidade do produto final. Os experts consideraram o conteúdo do material construído coerente com as necessidades do público-alvo. A organização do vídeo em diferentes passos proporcionou uma sequência lógica para a abordagem do conteúdo proposto.

Filmes e vídeos educativos devidamente construídos constituem-se ferramentas de suporte no processo de ensino aprendizagem. ${ }^{21} \mathrm{O}$ potencial de abrangência dos vídeos educativos pode ser explicado pela simplicidade dos recursos necessários à sua divulgação e acesso.

Nesse estudo, a linguagem utilizada no vídeo foi considerada adequada. $\mathrm{O}$ uso de linguagem acessível ao público-alvo é indispensável, uma vez que a falta de compreensão inviabiliza o alcance dos objetivos do material educativo. ${ }^{15,22}$

A abordagem de alguns procedimentos, como a mistura de insulina, mesmo com inúmeras tentativas de simplificação para favorecer a compreensão, constitui-se um desafio, por se tratar de um procedimento complexo. Estudo mostrou que apenas $34(48,6 \%)$ de $70(100,0 \%)$ pacientes, realizavam esse procedimento adequadamente. ${ }^{23}$ Foram ainda incorporadas sugestões dos experts referentes ao layout, aos textos, às imagens e às cores, todas com fundamentação na literatura. ${ }^{22,24}$

Nesse estudo, já no período de desenvolvimento, buscou-se estabelecer acordos com a equipe gestora local do sistema de saúde, visando garantir que o material desenvolvido fosse disponibilizado como material oficial no site do município. A existência de materiais educativos, por si só, não tem impacto sobre os resultados assistenciais. Eles precisam ser utilizados de maneira adequada pelas equipes na abordagem dos pacientes e de seus cuidadores. ${ }^{10}$

Salienta-se ainda que as pessoas a quem se destinam as ações educativas devem estar envolvidas no estabelecimento das necessidades de aprendizagem, no planejamento e na avaliação. ${ }^{25,26}$ Essa é uma limitação desse estudo, uma vez que a validação do material, ainda não foi realizada juntos aos pacientes e aos cuidadores.

Outra limitação é que o material produzido foi avaliado somente por profissionais com atuação na área da saúde. Ressalte-se ainda que a avaliação por profissionais de diferentes áreas, como letras, comunicação, som e imagem, poderia oferecer maior qualidade ao mesmo. ${ }^{15}$ Além disso, a avaliação foi realizada, em parte, a partir de um instrumento construído pelos próprios pesquisadores e, portanto, não validado.

Apesar dessas limitações, é importante apontar que a construção, avaliação e adequação do material produzido foi amparada pela prática profissional e traduz o anseio dos pesquisadores em aproximar pesquisa e prática, valorizando as potencialidades da complementariedade que existe nesse movimento.

\section{CONCLUSÃO E IMPLICAÇÕES PARA A PRÁTICA}

Quanto à construção, foi produzido um vídeo intitulado: "Aplicação de insulina: passo a passo", com tempo de duração de 08 minutos e 34 segundos, dividido em 13 passos, abrangendo os principais pontos críticos relacionados ao tema. Para a construção, foi elaborado um roteiro, contendo a descrição da imagem e do áudio.

Quanto à avaliação, o material foi submetido à apreciação de 10 experts ligados à gestão, assistência e/ou docência, em encontro presencial único. Os experts discutiram o material produzido e as sugestões resultantes foram registradas pela equipe de pesquisadores, além disso, utilizou-se um instrumento com afirmações e respostas tipo likert, elaborado pelos próprios pesquisadores.

A adequação foi realizada com base nos apontamentos realizados durante o processo de avaliação pelos experts e no instrumento de avaliação utilizado. Nessa fase, optou-se por produzir dois vídeos, sendo um sobre o processo de aplicação de insulina quando há prescrição de dois tipos de insulina e outro sobre o processo de aplicação quando apenas um tipo de insulina está prescrito. Para tanto, o roteiro construído na fase 1 foi alterado, gerando dois roteiros. A partir dos novos roteiros, foi realizada nova captação de áudio e vídeo, resultando na produção de dois vídeos.

Os vídeos educativos construídos podem ser considerados como uma ferramenta facilitadora do processo de educação em diabetes mellitus, útil na uniformização de orientações. 
A descrição do processo de construção, avaliação e adequação pode encorajar outros profissionais a desenvolver materiais que respondam às necessidades de seus contextos de trabalho, os quais poderão melhorar e qualificar o cuidado às pessoas.

\section{CONTRIBUIÇÃO DOS AUTORES}

Desenho do estudo. Aquisição, análise de dados e interpretação dos resultados. Redação e revisão crítica do manuscrito. Aprovação da versão final do artigo. Responsabilidade por todos os aspectos do conteúdo e a integridade do artigo publicado. Janaina Pereira da Silva. Gerson Alves Pereira Júnior.

Aquisição, análise de dados e interpretação dos resultados. Redação e revisão crítica do manuscrito. Aprovação da versão final do artigo. Responsabilidade por todos os aspectos do conteúdo e a integridade do artigo publicado. Filipe Andrade Bernardi. Leonardo Orlandin. Juliana Constantino Franzon. Gustavo Zanelatto Ferlin.

\section{EDITOR ASSOCIADO}

\author{
Ivone Evangelista Cabral
}

\section{REFERÊNCIAS}

1. American Diabetes Association. Introduction. Diabetes Care.2017;40(Suppl 1):S12. http://dx.doi.org/10.2337/dc17-S001. PMid:27979885.

2. Frid AH, Hirsch LJ, Menchior AR, Morel DR, Strauss KW. Worldwide injection technique questionnaire study. Mayo Clin Proc. 2016 set;91(9):1212-23. http://dx.doi.org/10.1016/j.mayocp.2016.06.011. PMid:27594185.

3. Sociedade Brasileira de Diabetes. Diretrizes da Sociedade Brasileira de Diabetes (2015-2016). São Paulo: A.C. Farmacêutica; 2016.

4. Takale AA, Behera AL, Banerje SK, Gadhave MV, Gaikawad DD. Insulin as an oral drug delivery: a novel approach. Int. J. Chem. Life Sci. 2012;2(2):1079-85.

5. Ferreira DSP, Daher DV, Teixeira ER, Rocha IJ. Repercussão emocional diante do diagnóstico de Diabetes Mellitus tipo 2. Rev enferm UERJ. [Internet]. 2013; [citado 2019 maio 19];21(1):41-6. Disponível em: https://www.e-publicacoes.uerj.br/index.php/enfermagemuerj/article/ view/6346/5885

6. Spollett G, Edelman SV, Mehner P, Walter C, Penfornis A. Improvement of insulin injection technique: examination of current issues and recommendations. Diabetes Educ. 2016 ago;42(4):379-94. http://dx.doi. org/10.1177/0145721716648017. PMid:27216036.

7. De Coninck C, Frid A, Gaspar R, Hicks D, Hirsch L, Kreugel G et al. Results and analysis of the 2008-2009 Insulin Injection Technique Questionnaire survey. J Diabetes. 2010;2(3):168-79. http://dx.doi org/10.1111/j.1753-0407.2010.00077.x. PMid:20923482.

8. Weaver RG, Hemmelgarn BR, Rabi DM, Sargious PM, Edwards AL, Manns BJ et al. Association between participation in a brief diabetes education programme and glycaemic control in adults with newly diagnosed diabetes. Diabet Med. 2014 dez;31(12):1610-4. http://dx.doi. org/10.1111/dme.12513. PMid:24890340.

9. Bell AM, Fonda SJ, Walker MS, SchmidtV, Vigersky RA. Mobile phone-based video messages for diabetes self-care support. J Diabetes Sci Technol. 2012 mar 1;6(2):310-9. http://dx.doi.org/10.1177/193229681200600214. PMid:22538140.

10. Fracolli $L A$, Chiesa AM. A percepção das famílias sobre a cartilha "toda hora é hora de cuidar". Mundo saúde (Impr.). 2010;34(1):36-42.
11. Gonçalves VM, Pacífico CF, Teixeira PG, Nagami V, Zazula R, Gon MCC. Análise dos materiais educativos sobre diabetes para crianças. Rev Perspect Psicol. [Internet]. 2014; [citado 2019 maio 19];18(1):44-56. Disponível em: http://www.seer.ufu.br/index.php/perspectivasempsicologia/ article/view/28576

12. Souza VL No, Monteiro AC, Lima AC, Ferreira AHR, Silva JC, Lima MA. Recurso áudio visual para aprendizagem sobre afogamento: relato de experiência. Rev Enferm UFPI. 2017;6(1):80-3. http://dx.doi. org/10.26694/reufpi.v6i1.5505.

13. Hazen RA, Eder M, Drotar D, Zyzanski S, Reynolds AE, Reynolds P et al A feasibility trial of a video intervention to improve informed consent for parents of children with leukemia. Pediatr Blood Cancer. 2010 jul;55(1):113-8. http://dx.doi.org/10.1002/pbc.22411. PMid:20063423.

14. Portaria $n^{\circ} \mathbf{2 . 5 8 3}$, de 10 de outubro de 2007 (BR). Define elenco de medicamentos e insumos disponibilizados pelo Sistema Único de Saúde, nos termos da Lei ํㅜ⒒347, de 2006, aos usuários portadores de diabetes mellitus. Diário Oficial da União [periódico na internet], Brasília (DF), 2007 [citado 2018 ago 23]. Disponível em: http://bvsms.saude.gov.br/bvs/ saudelegis/gm/2007/prt2583_10_10_2007.html\#: :text=PORTARIA\%20 N\%C2\%BA\%202.583\%2C\%20DE\%2010, usu\%C3\%A1 rios\%20 portadores $\% 20 \mathrm{de} \% 20$ diabetes $\% 20$ mellitus.\&text=Considerando $\% 20$ a\%20pactua\%C3\%A7\%C3\%A30\%20da\%20Comiss\%C3\%A3o,Art

15. Echer IC. Elaboração de manuais de orientação para o cuidado em saúde. Rev Latino-Am Enfermagem. 2005;13(5):754-7. http://dx.doi. org/10.1590/S0104-11692005000500022.

16. Ribeirão Preto (SP), Secretaria Municipal da Saúde. Protocolo de atendimento em hipertensão e diabetes. Ribeirão Preto: Secretaria Municipal da Saúde de Ribeirão Preto; 2011.

17. Alexandre NMC, Coluci MZO. Validade de conteúdo nos processos de construção e adaptação de Instrumentos de medidas. Ciênc. Saúde Colet (Barueri). 2011;16(7):3061-8. http://dx.doi.org/10.1590/S141381232011000800006.

18. Rosa BVC, Girardon-Perlini NMO, Gamboa NSG, Nietsche EA, Beuter A, Dalmolin A. Development and validation of audiovisual educational technology for families and people with colostomy by cancer. Texto Contexto Enferm. 2019;28:e20180053. http://dx.doi.org/10.1590/1980265x-tce-2018-0053.

19. Grassi G, Scuntero P, Trepiccioni R, Marubbi F, Strauss K. Optimizing insulin injection technique and its effect on blood glucose control. J Clin Transl Endocrinol. 2014 dez;4(1):145-50. http://dx.doi.org/10.1016/j. jcte.2014.07.006. PMid:29159095

20. Ministério da Saúde (BR). Estratégias para o cuidado da pessoa com doença crônica: diabetes mellitus. Brasília: Ministério da Saúde;2013.

21. Razera APR, Trettene AS, Mondini CCSD, Cintra FMRN, Tabaquim MLM. Educational video: a training strategy for caregivers of children with cleft lip and palate. Acta Paul Enferm. 2016 ago;29(4):430-8. https:/ doi.org/10.1590/1982-0194201600059.

22. Doak CC, Doak LG, Root JH. Teaching patients with low literacy skills $5^{a}$ ed. Philadelphia: J. B. Lippincott Company Philadelphia; 1996. http:// dx.doi.org/10.1097/00000446-199612000-00022.

23. Patil M, Sahoo J, Kamalanathan S, Selviambigapathy J, Balachandran $\mathrm{K}$, Kumar R et al. Assessment of insulin injection techniques among diabetes patients in a tertiary care centre. Diabetes Metab Syndr. 2017;11(Suppl 1):S53-6. http://dx.doi.org/10.1016/j.dsx.2016.09.010. PMid:27614866.

24. Farina $M$, Perez $C$, Bastos D. Psicodinâmica das cores em comunicação. $5^{\underline{a}}$ ed. São Paulo: Edgard Blucher; 2006.

25. Manoel MF, Marcon SS, Baldissera VDA. Estratégias educativas para pessoas com hipertensão arterial e diabetes mellitus. Rev Enferm UERJ. [Internet]. 2013; [citado 2019 maio 19];21(3):403-8. Disponíve em: https://www.e-publicacoes.uerj.br/index.php/enfermagemuerj/ article/view/7551

26. Razera APR, Trettene AS, Mondini CCSD, Cintra FMRN, Razera FPM, Tabaquim MLM. Construction of an educational video on postoperative care for cheiloplasty and palatoplasty. Texto Contexto Enferm. 2019;28:e20180301. http://dx.doi.org/10.1590/1980-265xtce-2018-0301. 